\title{
Emociones y trabajo docente: organizando un debate científico ausente en América Latina ${ }^{1}$
}

\author{
Rodrigo Cornejo Chávez \\ Gabriel Etcheberrigaray \\ Sebastián Vargas \\ Jenny Assaél \\ Universidad de Chile
}

\begin{abstract}
Resumen
El presente texto tiene por objetivo presentar una revisión de literatura empírica respecto de la investigación contemporánea sobre las emociones y el trabajo docente. Se revisaron las bases de datos WOS y Scopus hasta el año 2018. Se revisó la literatura y se organizó la misma en base a los principales tópicos de investigación y los hallazgos más importantes al interior de cada uno de ellos. Los tópicos identificados fueron siete: 1) el estudio de las “emociones discretas" en la docencia; 2) emociones, burnout, malestar y agotamiento emocional entre profesores/as; 3) las emociones en la identidad docente y el desarrollo profesional; 4) emociones docentes y relaciones con otros actores educativos; 5) emociones docentes en contextos históricos, políticos y sociales, y de reformas educativas; 6) regulaciones y labor emocional; 7) diferencias contextuales y personales en las experiencias emocionales docentes. Asimismo, se identificó una temática transversal que aún no consigue abordarse de manera sistemática en la literatura y que tiene que ver con la naturaleza del saber o conocimiento emocional que construyen los/as docentes. Finalmente se discuten las posibilidades y aportes de una agenda de investigación sobre la temáticas en América Latina. Palabras clave: Emociones y docencia; trabajo emocional docente; revisión de literatura
\end{abstract}

\section{Resumo}

O texto objetiva apresentar uma revisão da literatura empírica a respeito da pesquisa contemporânea sobre as emoções e o trabalho docente. Revisaram-se as bases de dados WOS e Scopus até o ano 2018. Ademais, foi revisada e organizada a literatura de acordo com os principais tópicos de pesquisa. Os tópicos identificados foram sete: 1) o estudo das “emoções discretas” na docência; 2) emoções, burnout, mal-estar e esgotamento emocional entre professores; 3) as emoções na identidade docente e o desenvolvimento profissional; 4) emoções docentes e relações com outros atores educacionais; 5) emoções docentes em contextos históricos, políticos e sociais, junto com reformas educacionais; 6) regulações das emoções e "labor emocional”; 7) diferenças contextuais e pessoais nas experiências emocionais docentes. Da mesma forma, identificou-se uma temática transversa ainda não abordada de modo sistemático na literatura, que tem a ver com a natureza do saber ou conhecimento emocional dos docentes. Por fim, discutem-se as possibilidades e possíveis aportes de uma agenda de pesquisa a temática na América Latina.

Palavras-chave: Emoções e trabalho docente; trabalho emocional docente; revisão integrativa

\begin{abstract}
This present text aims to present an empirical literature review on contemporary research on emotions and teachers' work. We searched WOS and Scopus databases up until the year 2018. The reviewed literature was organized around the main research topics and most relevant findings in each of them. The topics identified were seven: 1) The study of "discrete emotions" in teaching; 2) emotions, burnout, malaise and emotional exhaustion among teachers; 3) emotions in teacher identity and professional development; 5) teachers' emotions and relationships with other educational actors; 5) teachers' emotions within historical, political and social contexts and educational reforms; 6) regulations and emotional labor; and 7) personal and contextual differences in teachers' emotional experiences. Additionally, the topic of the nature of teachers' emotional knowledge or wisdom was identified as present across the reviewed literature, albeit not approached in a systematic manner. Finally, there is a discussion on the possibilities and possible contributions towards a research agenda on emotions and teachers' work in Latin America.
\end{abstract}

Keywords: Emotions and teaching, teachers' emotional work, literature review

ISSN 1645-1384 (online) www.curriculosemfronteiras.org 


\section{Introducción}

La labor de los docentes es un factor clave, y crítico a la vez, en los múltiples esfuerzos que se realizan, en América Latina y el mundo entero, para mejorar la pertinencia y calidad de los aprendizajes escolares y desarrollar herramientas para la vida ciudadana de los estudiantes (Schleicher, 2016; UNESCO, 2015; Schwartz; Wurtzel; Olson, 2007; Fullan, 2003). Sin embargo, pese a la gran cantidad de conocimiento generado respecto de la labor docente, diversos estudios internacionales señalan que aspectos cruciales de esta, como las condiciones sociales de la docencia, la evaluación, la disposición emocional y la competencia profesional, siguen sin resolverse (Schleicher, 2016; Poggi, 2013). Diversos investigadores han manifestado que las características propias del oficio docente obligan a complejizar los marcos referenciales y metodológicos para acercarse al trabajo real, complejo y situado que realizan los profesores (Poggi, 2013; Comber \& Nixon, 2009; Hargreaves, 1997).

La revisión de literatura empírica que presentamos a continuación aspira a aportar conocimiento sobre uno de los aspectos más complejos e invisibilizados de la labor docente: su naturaleza emocional. Los aspectos emocionales son centrales en los procesos reales de trabajo docente, los que suelen quedar invisibilizados por las regulaciones oficiales del trabajo, por las agendas de investigación y por los/as propios/as trabajadores/as docentes (Cornejo, 2018). Como veremos más adelante, estos estudios tienen un desarrollo de apenas un par de décadas en Europa, USA y algunos países del Sudeste asiático.

Estos aspectos emocionales se sitúan en el centro de lo que Dejours (2015) denominó la brecha entre la organización prescrita del trabajo y las actividades laborales realmente realizadas. Para este autor, esta brecha genera malestar y sinsentido, y frente a ella el trabajador, el/la docente en este caso, se ve forzado/a a desarrollar una capacidad individual y colectiva para enfrentar esta brecha entre lo prescrito y lo real, y resolver los múltiples desafíos que le impone el trabajo cotidiano, lleno de actividades no previstas en la organización prescrita del trabajo.

No es de extrañar, entonces, que los aspectos emocionales del trabajo docente hayan sido muy poco estudiados por las ciencias sociales y de la educación. Aquí radica la pertinencia de este trabajo, que se propone presentar una panorámica de las investigaciones sobre emociones y trabajo docente, con el objetivo de ayudar a abrir una agenda de investigación sobre la temática en América Latina. Para ello, revisamos las bases de datos WOS y Scopus hasta el año 2018, así como los libros y handbooks que aparecían más citados en la literatura revisada.

Comenzaremos este texto, dando cuenta del estado actual de la investigación sobre emociones y trabajo docente. Posteriormente organizaremos y resumiremos los principales hallazgos de la literatura especializada sobre emociones en la docencia. En tercer lugar, nos referiremos a una temática crítica que la literatura no ha logrado organizar aún: el saber emocional docente. Por último, finalizaremos este texto, desarrollando brevemente los principales desafíos para la investigación del trabajo emocional docente desde América Latina. 


\section{El estudio de las emociones en educación y en el trabajo docente: el giro afectivo}

Si bien la importancia de las emociones en los procesos educativos está documentada conceptualmente hace décadas (Vigotsky, 1998), los estudios empíricos sobre esta temática son recientes y, en el caso específico de las emociones docentes, no tienen más de dos décadas de desarrollo (Uitto; Jokikkoko; Estola, 2015; Fried; Mansfield; Dobozy, 2015; Sutton; Wheatly, 2003). Pekrun y Linnenbrink-Garcia (2014) sostienen que desde la segunda mitad de la década de 1990 las ciencias sociales y de la educación están experimentando un giro afectivo. Este reciente interés en las emociones ha dado lugar a un número creciente de estudios empíricos sobre emociones en el trabajo docente, provenientes de diversas disciplinas: psicología educacional, psicología social, sociología de la educación, antropología e investigación sobre profesores y enseñanza (Sutton; Wheatly, 2003). Esta proliferación de estudios ha hecho posible, recientemente, la elaboración de al menos siete revisiones sistemáticas de evidencia empírica sobre emociones docentes (Uitto; Jokikokko; Estola, 2015; Fried; Mansfield; Dobozy, 2015; Ye; Chen, 2015; Saric, 2015; Tsang, 2014; Keller; Frenzel; Goetz; Pekrun; Hensley, 2014; Sutton; Wheatly, 2003), la edición de dos libros recopilatorios de evidencias y enfoques metodológicos sobre emociones y educación (Zembylas; Schutz, 2016, 2009) y la publicación del primer International Handbook de emociones en educación (Pekrun; Linnenbrink-Garcia, 2014).

Uno de los grandes problemas o déficits de los estudios sobre emociones en educación y, particularmente en el trabajo docente, es la falta de acuerdo respecto a cómo deben ser conceptualizadas las emociones. Zembylas (2007a), basado en una revisión de la literatura, distingue tres diferentes aproximaciones teóricas al estudio de las emociones en la educación: 1) enfoques psicodinámicos [que] reconocen las emociones como fenómenos privados, individuales y fisiológicos; 2) enfoques socio construccionistas [que] ven las emociones determinadas principalmente por las relaciones y contextos sociales; y 3) los enfoques interaccionistas (especialmente teorías feministas y post estructuralistas), [que] ponen de relieve las emociones no sólo como cargadas de lenguaje, sino como encarnadas y performativas" (Zembylas, 2007a, p.357).

Desde las ciencias psicológicas, si bien no hay un único enfoque aceptado sobre las emociones y los procesos emocionales, es posible identificar ciertos acuerdos básicos. En primer lugar, la idea de la emoción como fenómeno multicomponencial (Sutton; Weathly, 2003). Al respecto, Sutton y Weathly (2003) señalan que los principales componentes de una emoción serían: a) una evaluación o juicio de la situación social o personal, b) una experiencia subjetiva, c) un conjunto de cambios fisiológicos, d) una necesidad de expresar (que no siempre se despliega) y e) una orientación hacia la acción. En segundo lugar, la idea de que la valencia y la intensidad serían los rasgos que definen la experiencia emocional; entendiendo por valencia la valoración en términos de agrado y desagrado de la emoción, y 
por intensidad el nivel de activación corporal involucrado (Pekrun, 2014). En tercer lugar, la distinción entre rasgos emocionales y estados emocionales. Se entiende por rasgo emocional la propensión de los profesores a experimentar una emoción en particular en el contexto de la enseñanza, asumiéndose que es un atributo relativamente estable de los profesores a través del tiempo. Los estados emocionales, en cambio, capturan el estado emocional específico de la situación y varía dependiendo de las condiciones contextuales [en este caso las condiciones de trabajo docente] (Keller; Frenzel; Goetz; Pekrun; Hensley, 2014). Los estudios sobre "emociones discretas" a los que nos referiremos luego, tienen que ver con esta distinción. En cuarto lugar, el énfasis en el estudio de la construcción intra psicológica de las emociones ha devenido en la línea de investigación sobre regulación emocional, lo que desarrollamos más adelante (Saric, 2015).

Desde las perspectivas socio construccionistas e interaccionistas se plantea que las emociones "se ubican no sólo en las mentes individuales, [sino que] están impregnadas y expresadas en las interacciones y relaciones humanas” (Hargreaves, 2000, p.824). De tal forma que las emociones no son solo "asuntos privados concernientes a disposiciones personales o cualidades psicológicas, sino además [son] experiencias políticas y sociales que están construidas como un escenario de trabajo, en este caso la docencia” (Zembylas, 2003, p.216). Varios investigadores enfatizan en la importancia de las dimensiones de poder para comprender las emociones docentes, no solo en la dimensión macro social, sino en las relaciones que se establecen al interior de la escuela y la sala de clases (Tsang, 2014; Zembylas, 2005). Por otra parte, si bien muchos estudios sociológicos han centrado su mirada en las transformaciones en las regulaciones educativas y los procesos de trabajo para comprender las emociones docentes (Carter; Stevenson, 2012), en ellos se plantea que las emociones deben ser entendidas como mediadoras entre los procesos macro y micro sociales (Tsang, 2014), o como performances transaccionales en el espacio liminal entre lo individual y lo social (Zembylas; Charalambous; Charalambous, 2012). En ese sentido, "la capacidad de las personas para usar sus emociones adecuadamente en el lugar de trabajo depende de dos elementos, además de la competencia emocional individual; [de] lo que se espere emocionalmente del trabajo o la profesión de las personas, y cómo su organización estructure las interacciones humanas en formas que ayuden o dificulten la expresión emocional y la comprensión” (Hargreaves, 2000, p.825). Siguiendo esta línea argumental, varios autores enfatizan en la imbricada relación entre emociones, juicios sociales e intencionalidades o sentidos de las personas. Estos juicios emocionales, no son solamente reacciones frente al mundo, sino formas subjetivas de involucrarse en éste. "Las emociones están, por lo tanto, imbricadas en la historia, son lógicas sociales, culturales y políticas y tienen propósitos y sentidos, implicando nuestras visiones del futuro y lo que nosotros consideramos como vivir bien. Hablan al tipo de personas que somos, a nuestra identidad, y por lo tanto entre ellas están enredadas nuestras esperanzas, expectativas y deseos” (Bullough, 2009, p. 36).

Estos dos gruesos enfoques para el estudio de las emociones en el trabajo docente (los que se centran en los procesos intrapersonales y los que se centran en procesos sociales y culturales) han comenzado recientemente a dialogar. Son varios los autores que se abocan a 
comprender las complejas relaciones existentes entre las condiciones estructurales de trabajo y los procesos de agenciamiento individual y colectivo en las emociones docentes (Tsang, 2014, Zembylas; Charalambous; Charalambous, 2012). Retomaremos este debate hacia el final de este texto.

\section{Las emociones en el trabajo docente: ¿Qué dice la evidencia?}

Los estudios de emociones docentes utilizan una variedad de enfoques metodológicos (Saric, 2015; Uitto; Jokikokko; Estola, 2015). La evidencia acumulada es diversa y no existe una única forma de organizarla (de hecho, las revisiones de evidencia estudiadas no presentan acuerdo al respecto). Para este apartado, la hemos organizado en torno a 7 tópicos de investigación, con el fin de obtener una panorámica general actualizada, aunque sin duda hay estudios que podrían situarse en más de un tópico:

2.1. El estudio de las "emociones discretas” en la docencia: según Frenzel (2014) se trata de la temática en la cual hay más evidencia relativa acumulada, aun cuando esta sigue siendo escasa. Esta autora señala que las emociones discretas (o específicas) positivas que aparecen reportadas en más estudios son: el placer, el orgullo y la compasión. Uitto et al (2015) añaden en su revisión el amor y el cuidado hacia los estudiantes como emociones de valencia positiva, así como la satisfacción. La mayoría de los estudios señalan que las emociones docentes de valencia positiva están directamente vinculadas con la cualidad de la relación con sus estudiantes y con la evaluación de la posibilidad de conseguir logros educativos en la interacción de aula. En términos negativos, las emociones reportadas en más estudios son el enojo, la ansiedad, el complejo vergüenza/culpa y el aburrimiento (Frenzel, 2014). Estas suelen aparecer vinculadas a mal comportamiento e indiferencia de parte de los estudiantes. Aunque la ansiedad ha sido más vinculada a la incertidumbre respecto a los cambios sociales, culturales y políticos en curso y a periodos de implementación de reformas educativas centradas en la estandarización, que conllevan a procesos de intensificación laboral (Saric, 2015; Tsang, 2014; Kelchtermans, 2005). Uitto et al (2015) destacan la presencia de la pena, vinculada al trabajo solitario y aislado que perciben algunos docentes. Frenzel, Becker-Kurz, Pekrun y Goetz (2015) señalan que los docentes reportan más emociones de valencia positiva que negativa, a lo que Zembylas (2005) agrega que para los docentes es más difícil y lento salir de los estados emocionales negativos que de los positivos. Por otra parte, varios autores se han dedicado a estudiar el rol de los procesos cognitivos personales en la generación de emociones docentes (Frenzel et al 2015; Mevarech; Maskit, 2015). El debate actual se centra en intentar comprender la medida en que las emociones son influidas por una disposición personal (cognitiva) a experimentar dichos estados y la medida en que son generadas o, al 
menos, propiciadas por el contexto.

2.2. Emociones, burnout, malestar y agotamiento emocional entre profesores: la tradición de investigación sobre burnout y malestar es la más antigua y nutrida de las que se revisaron. La mayoría de estas investigaciones no se han realizado desde enfoques educativos, sino más bien clínicos o de salud ocupacional (Frenzel, 2014). El burnout es descrito como un síndrome, es decir, un conjunto de síntomas asociados a factores de la organización del trabajo, los que también se asocian a enfermedades docentes (Cornejo, 2009; UNESCO, 2005; Kyriacou, 2001). Las condiciones de trabajo que se constituyen en factores de riesgo para la salud docente son, según la investigación, aquellos denominados factores psicosociales del trabajo, entre los que destacan: carga de trabajo, exigencias psicológicas, pérdida de sentido en el trabajo, falta de apoyo social, pérdida de autonomía y falta de apoyo para lidiar con las demandas del hogar (UNESCO, 2005; SUSESO, 2010, Cornejo, 2009; Van der Doef ; Maes, 1999).

2.3 Las emociones en la identidad docente y el desarrollo profesional: se trata en general de estudios realizados con enfoques socio construccionistas e interaccionales. Estos vinculan la experiencia emocional docente con los sentidos profesionales y las creencias sobre su rol (Uitto et al, 2015). O'Connor (2008) señala que las emociones docentes están mediadas por "acciones y reflexiones que resultan de un deseo de los docentes a motivar, ayudar o inspirar a sus alumnos" (O'Connor, 2008, p. 117). Por su parte, Newberry (2010) señala que el cuidado es una motivación para seguir en la docencia, pero a la vez una demanda que puede resultar agotadora, pues el cuidado no existe automáticamente en la enseñanza, sino que requiere labor emocional (ver más adelante). Para algunos autores es importante distinguir ente el “cuidado ético” que está prescrito y requiere de una intensa labor emocional de parte de los docentes, y el "cuidado natural" que emerge cuando los docentes lo ven como parte intrínseca y natural del trabajo (Newberry, 2010; Zembylas, 2007)

2.4. Emociones docentes y relaciones con otros actores: existe acuerdo entre los investigadores respecto de que las relaciones interpersonales más intensas reportadas por los docentes son las relaciones que construyen con sus estudiantes, por sobre las relaciones con sus colegas, sus directivos y los padres y madres. Estas relaciones generan múltiples emociones en los profesores, las que a su vez, condicionan las mismas relaciones interpersonales (Saric, 2015; Uitto et al, 2015). Señalábamos más arriba que las emociones docentes de valencia negativa están relacionadas con elementos actitudinales de los estudiantes. También se ha reportado que los profesores influyen en los estudiantes haciéndolos sentir tristes o avergonzados cuando les expresan emociones negativas, o bien, aumentando su tendencia a colaborar y seguir las normas de aula cuando les expresan emociones positivas como cuidado y preocupación (Sutton; Wheatley, 2003). Saric (2015) señala que las emociones docentes tienen impacto en los procesos cognitivos y emocionales 
de los estudiantes, así como en sus resultados académicos y conductuales. Asimismo, se ha reportado que los estudiantes son conscientes de las emociones que experimentan sus profesores. Por último, se han reportado entre profesores y estudiantes fenómenos de transferencia y confusión emocional que influencian sus relaciones (Saric, 2015; Hagenauer; Hascher; Volet, 2015)

2.5 Emociones docentes en contextos históricos, políticos y sociales, y reformas educativas: Existe nutrida evidencia respecto al impacto de ciertas políticas educativas en las emociones de los docentes. Fried, Mansfield y Dobozy (2015) señalan que las políticas de rendición de cuentas y evaluaciones de altas consecuencias han cambiado la naturaleza de las interacciones en el aula, impactando la relación de estudiantes y profesores e incrementando el desgaste de estos últimos. Tsang (2014) señala que la intensificación laboral derivada de las nuevas políticas educativas se vincula a emociones de culpa, ansiedad y pérdida de sentido. Otros autores señalan que las políticas que aumentan la presión por resultados generan ansiedad y agotamiento emocional (Chen, 2016; Tsang; Kong, 2016). Tsang y Kong (2016) enfatizan en los efectos emocionales negativos del incremento de tareas que son percibidas por los docentes como no instruccionales (vinculadas a generación de evidencias, registros y papeleos). Por otra parte, algunos autores señalan que si no se abordan estas consecuencias emocionales, los proyectos de reforma pueden ser saboteados (Kelchtermans, 2005; Lasky, 2005),

2.6. Regulaciones y labor emocional: se trata de uno de los debates más intensos de la investigación sobre emociones docentes (Lee et al, 2016; Ye; Chen, 2015). La categoría "regulación emocional" surge desde las ciencias psicológicas y se refiere al proceso a través del cual los individuos influyen en qué emociones tienen, cuándo las tienen, cómo las experimentan y cómo las expresan a otros (Saric, 2015). Desde esta teoría se describen las estrategias personales que ocurren en distintos momentos del proceso emocional para aumentar las emociones de valencia positiva y disminuir las de valencia negativa (Pekrun, 2014). Desde un enfoque cognitivo, Saric (2015) define 5 familias de estrategias de regulación: selección y modificación de situación (por ej. cuando el docente a través de la planificación anticipa una situación de aula), manejo de la atención (por ej. ignorar conductas disruptivas), reevaluación cognitiva (por ej. buscar explicaciones alternativas a las acciones de los estudiantes) y supresión directa de emociones (por ej. contener expresiones de ira o pena). Por su parte la "labor emocional” es una categoría introducida desde la sociología en los trabajos de Hochschild (1983) para referirse a la necesidad que tienen los trabajadores de manejar sus emociones y performance para alcanzar las exigencias que le hace la organización en cuanto a expresión y lenguaje corporal emocional. Las exigencias de la organización se operativizan en un conjunto de reglas de expresión emocional. En el caso de la docencia se han descrito tres fenómenos al respecto: “1) las reglas de expresión emocional de los docentes no solo ocurren en la sala de clases, sino también después de clases; 2) estas reglas son distintas según el objetivo de sus interacciones, tales como estudiantes, padres, 
colegas y otras personas; 3) la expresión emocional del docente requiere controlar y restringir las emociones negativas para poder cumplir su objetivo” (Ye \& Chen, 2015, p. 2234). Para Zembylas (2005), las reglas de expresión emocional para los docentes pueden resumirse en que los profesores no debieran expresar emociones de forma demasiado intensa o débil. La exigencia de cumplir estas "reglas de expresión emocional” obligan a los docentes a recurrir a ciertas "estrategias de labor emocional" (Hochschild, 1983). Se han descrito cuatro estrategias de labor emocional: 1) la actuación superficial, que se da cuando la sensación individual es diferente de la emoción que es requerida por la organización y el trabajador no ajusta su sensación interior, sino que realiza el comportamiento emocional externo requerido; 2) la actuación profunda, que se da cuando, frente a la inconsistencia entre las sensaciones individuales y los requerimientos organizacionales para la expresión de emociones, los trabajadores cambian internamente las emociones sentidas, alineándolas con los requerimientos organizacionales de expresión emocional; 3) la expresión de las emociones naturalmente sentidas, que se da cuando las sensaciones internas emocionales y los requisitos de la organización para el desempeño emocional son consistentes, y los trabajadores reflejan sus emociones tal como las sienten, 4) la acción de disonancia deliberativa, que se da cuando existe un requerimiento de sensación emocional y un requerimiento de expresión emocional y ambos provienen de la organización y son inconsistentes (Ye; Chen, 2015; Tsang, 2014). Diversos estudios plantean que la estrategia más utilizada por los docentes es la expresión de emociones naturalmente sentidas, seguida por alguno de los tipos de actuación, aunque la mayoría de los docentes combina estrategias de labor emocional (Ye; Chen, 2015). Por otra parte, la evidencia señala que la actuación superficial sostenida en el tiempo genera procesos de agotamiento emocional en los docentes (Zembylas, 2005). La discusión sobre los alcances de la "regulación emocional" y la "labor emocional” da cuenta de las complejidades de un fenómeno que es individual, cultural y social a la vez. Para algunos autores lo coherente sería avanzar, desde la evidencia empírica, hacia una integración de ambas teorías en la docencia, pues no resulta consistente desconocer las reglas de expresión emocional que existen en la docencia, así como las genuinas emociones de amor y cuidado natural que sienten los docentes hacia sus estudiantes (Lee et al, 2016).

2.7. Diferencias contextuales y personales en las experiencias emocionales docentes: si bien la literatura revisada no es muy enfática, se reportan diferentes experiencias emocionales vinculadas al género de los docentes y a su experiencia profesional. Las docentes mujeres parecen ser más propensas a desarrollar estrategias de actuación profunda, modificando sus emociones de acuerdo a los requerimientos institucionales, a la vez que tienden a utilizar más la expresión de emociones naturalmente sentidas, en tanto que los varones parecen ser más propensos a la utilización de estrategias de actuación superficial (Ye; Chen, 2015). Por otra parte está descrito que los docentes nuevos suelen presentar mayores niveles de ansiedad que los docentes con mayor experiencia, los que parecen ser más favorables a la actuación profunda (Ye; Chen, 2015). Por otra parte, la intensidad de las emociones docentes parece disminuir hacia el final del ciclo laboral (Mevarech; Maskit, 2015). En el plano contextual, 
emergen dos dimensiones en las cuales se discute acerca de las particularidades de la experiencia emocional docente. En primer lugar, el nivel de vulnerabilidad de la población estudiantil: diversos estudios señalan que la experiencia emocional de los docentes es más intensa, agotadora y confusa en estos contextos (Cornejo, 2018; Little; Bartlett, 2010). En segundo lugar, el nivel educativo: los clásicos estudios de Hargreaves $(1998,2000)$ señalaban que las aulas de la escuela primaria eran sitios emocionalmente más intensos que las de secundaria para los docentes. Sin embargo, estudios más recientes señalan que la intensidad emocional de la relaciones educativas en la enseñanza secundaria no solo no es menor, sino que se despliega de manera menos predecible y más disruptiva para los docentes (Lasky, 2005; Cornejo, 2018).

\section{El saber emocional en el trabajo docente}

Dada la naturaleza del fenómeno de las emociones docentes, que presenta una enorme variabilidad, dependiendo de los juicios, sentidos e interacciones de los sujetos, cobra particular importancia la temática del saber profesional sobre la actividad emocional en el trabajo. Si bien existen diversos enfoques para conceptualizar este saber docente (capacidades, competencias, conocimientos, inteligencias), es el enfoque del saber docente propuesto por Shulman (1987) el que ha permitido el desarrollo posterior de la categoría Saber emocional. Para Schulman (1987), el saber docente es una amalgama de contenido y pedagogía que es particular al campo de los/as docentes. Zembylas (2007a) sostiene que el saber docente "es una forma de ecología de saber, un sistema que consiste en diversas fuentes y formas de conocimiento en una relación simbiótica” (Zembylas, 2007, p. 356), planteando que el saber emocional de un docente acerca de enseñar y aprender es parte indisoluble del ecosistema del saber docente, que él denomina ecología emocional (Zembyas, 2007a). Esta ecología emocional es el "saber emocional de un docente en un contexto social y político particular, incluyendo las abundantes conexiones a experiencias emocionales, y relaciones con otros/as a lo largo del tiempo" (Zembylas, 2007a, p. 357). Para poder conducir el proceso educativo, los docentes deben ser capaces de conectar su entendimiento emocional con lo que saben acerca de la asignatura, la pedagogía, los discursos de la escuela, las historias personales y el currículum. Esta propuesta de saber emocional se alinea con la tradición que enfatiza el carácter experiencial del saber docente. Para Rockwell (2011) el saber docente corresponde a los conocimientos o saberes que surgen a través de la experiencia del ejercicio de la docencia, de la trayectoria laboral e historia personal, y les permite a los docentes anticipar e improvisar lo que tienen que hacer en su trabajo cotidiano. En esa misma línea, Tardif (2004) señala que este saber, que es plural y social, profesional y experiencial, colectivo e individual, no tiene su origen en las instituciones de formación docente, sino que en la práctica de la profesión (Tardif, 2004), lo que plantea enormes desafíos para abordar el ámbito emocional en la formación inicial docente. Tal como señala Meyer (2009) un 
componente crítico de la formación docente es "la atención a los aspectos situados de la enseñanza, estando el foco puesto en los aspectos prototípicos de la pedagogía, casi sin considerar las experiencias emocionales del aula y la cultura profesional que sustenta estas experiencias” (Meyer, 2009, p.75). La vivencia de las primeras experiencias emocionales en el aula suele constituir un fuerte impacto para los docentes noveles.

\section{Desafíos para la investigación actual desde América Latina}

Como hemos visto en esta revisión panorámica, las emociones en el trabajo docente son un campo problemático de la realidad social educativa, que excede el estado actual del desarrollo teórico metodológico (Zembylas; Schutz, 2016). Los investigadores dedicados a la temática plantean el desafío de complejizar las teorías y enfoques para el estudio de las emociones docentes para que”...abarquen la naturaleza multi compuesta, dinámica y contextualizada de las emociones“(Fried et al, 2015, p.433). El incipiente diálogo que referíamos más arriba entre los enfoques intra psicológicos y sociales para el estudio de las emociones docentes, puede entenderse como parte de este desafío. Este diálogo intenta captar la tensión dinámica permanente que existe entre las condiciones sociolaborales y los agenciamientos individuales y colectivos en las emociones docentes (Tsang, 2014) y ha dado lugar a la creación de algunos modelos conceptuales integradores, tales como el modelo de ecología emocional (Zembylas, 2007a) y el modelo de Fried et al (2015) que intenta articular procesos intra psicológicos, interpersonales, de expresión emocional, evaluaciones de contexto, funciones emocionales y factores sociales culturales y políticos (Fried et al, 2015). Se trata de modelos conceptuales en proceso, construidos para captar la complejidad del fenómeno y orientar el desarrollo de futuras investigaciones, más que dar explicaciones acabadas del mismo. En los enfoques y modelos actuales se plantea la necesidad de "desafiar la fuerte dicotomía entre la emoción (no racional) y la razón (racional)” (Uitto et al, 2015, p. 9), así como abordar la relación imbricada entre propósitos educativos, contextos y experiencia emocional docente (Tsang, 2014).

En un plano epistemológico, en el desafío de construir conocimiento acerca de las emociones docentes, se pone de manifiesto la tensión entre las dificultades para construir un saber científico sistematizado y las complejidades que tienen los sujetos mismos para acceder y relacionarse con sus propias emociones. Para Saric (2015) un tema fundamental para la generación de conocimiento es fomentar la evaluación reflexiva de las propias experiencias emocionales de los profesores, así como una creciente conciencia emocional para responder adecuadamente en situaciones emocionales en el aula. Otros autores señalan el elevado riesgo que se corre cuando se construyen distinciones y análisis de la experiencia emocional sin incorporar la voz de los mismos docentes (Uitto et al, 2015). En el plano metodológico, los investigadores sugieren articular diversos dispositivos de producción de datos, que apunten hacia diferentes dimensiones del fenómeno de las emociones docentes (personales, situacionales, políticas, culturales, etc.) (Zembylas, 2007b). En términos más específicos, 
diversos autores sugieren el uso de métodos que sean consistentes con la naturaleza de la vivencia emocional que es individual, única y absolutamente contextualizada; tales como estudios de caso, la combinación de observaciones y entrevistas en profundidad; así también se sugiere el uso de enfoques de análisis de datos que permitan conceptualizar la propia experiencia de los docentes, tales como la teoría empíricamente fundamentada (Saric, 2015; Fried et al, 2015, Tsang, 2014).

Por otra parte, los autores señalan dos vacíos en el estado de la investigación actual sobre emociones docentes. Por un lado, un déficit de "transferencia de los resultados de la investigación de manera significativa en la formulación de políticas educativas” (Uitto et al, 2015, p.11), y de propuestas para la formación de futuros docentes (Frenzel, 2014). Por otro lado, la “...unilateralidad de contextos culturales en las investigaciones” (Uitto et al, 2015, p.12), pues no hay estudios provenientes de África ni Sudamérica. En Chile, si bien el tema comienza a cobrar mayor importancia, existe un escaso desarrollo de éste, centrado en propuestas y conceptualizaciones de orden clínico o filosófico aplicadas al ámbito educacional (e.g. Marchant, Milici \& Álamos, 2013, Naranjo, 2007; Casassus, 2007; Maturana, 1990).

Construir una agenda de investigación sobre emociones en el trabajo docente, o más específicamente sobre las dimensiones emocionales del trabajo docente en América Latina, resulta un asunto de la mayor urgencia. Como señalábamos al comienzo del texto, los aspectos emocionales son centrales en los procesos reales de trabajo docente y suelen ser difíciles de mirar y pensar. Con lo cual quedan circunscritos al ámbito del malestar y el sufrimiento corporal, o de lo anecdótico informal en el trabajo. Generar esta agenda de investigación puede ser un aporte para la reflexión colectiva sobre los aspectos emocionales del trabajo docente real. Los/as investigadores/as latinoamericanos podemos aprovechar la experiencia de dos décadas de investigación en otras regiones del mundo, repensar las problemáticas ya detectadas y evidencias generadas, situándolas en los contextos específicos de trabajo docente en América Latina. Asimismo, nos parece relevante, aprovechar la tradición que existe en nuestra región respecto a los estudios del trabajo docente. La categoría Trabajo, permite enfocar el fenómeno de la docencia desde una apertura a lo históricocultural, a lo complejo, a la realidad como construcción social. Pensar la docencia como trabajo permite referirla a la totalidad social, “totalidad que cumple una función gnoseológica importante, no solamente como crítica a la división de los campos disciplinarios, sino como fundamento para influir sobre la realidad” (Zemelman, 1992a, p. 32). Esperamos que este texto sea un aporte en ese sentido.

\footnotetext{
Notas

${ }^{1}$ Este trabajo fue realizado gracias al apoyo de la Agencia Nacional de Investigación y Desarrollo de Chile, a través de su proyecto FONDECYT N¹180801: “Dimensiones emocionales del trabajo docente: regulaciones, saberes y experiencias”.
} 


\section{Referencias Bibliográficas}

ALBORNOZ, Natalia; CORNEJO, Rodrigo Y ASSAÉL, Jenny. Condicionantes estructurales del vínculo entre profesor y estudiante: un análisis de los discursos docentes en el contexto actual de reforma educativa en Chile. Forum Qualitative Sozialforschung/Forum Qualitative Social Research FQS, 18 (3), Art. 15. DOI: http://dx.doi.org/10.17169/fqs-18.3.2632. 2017.

BULLOUGH, Robert. Seeking Eudamonia: The emotions in learning to teach and to mentor. In ZEMBYLAS, Michalinos; SCHUTZ, Paul. (Orgs.), Advances in teacher emotion research. The impact on teachers'lives. London: Springer, 2009 p. 33-54,

CARTER, Bob; STEVENSON, Howard. Teachers, workforce remodelling and the challenge to labour process analysis. Work, employment and society, v.26, n. 3, p. 481-496, 2012.

CASASSUS, Juan. La educación del ser emocional. Santiago: Índigo-Cuarto Propio. 2007.

CHEN, Junjun. Understanding teacher emotions: The development of a teacher emotion inventory. Teaching and Teacher Education, v. 55, p. 68-77, 2016

COMBER, Barbara; NIXON, Helen. Teacher's work and pedagogy in an era of accountability. Discourse: Studies in the cultural politics of education, v. 30, n. 3, p. 333-345, 2009

CORNEJO, Rodrigo. Repensar el trabajo docente en el Chile actual: una necesidad para la psicología educacional. En LEAL, Francisco (coord.) “Temas en Psicología Educacional: contribuciones para la formación de especialidad. Vol. I: Procesos y actores: claves para el asesoramiento educativo”. Buenos Aires: NOVEDUC. Pp. 263-296. 2018.

CORNEJO, Rodrigo. Condiciones de trabajo y bienestar/malestar docente en profesores de enseñanza media de Santiago de Chile. Educação \& Sociedade, 30(107), 2009.

DE SOUSA SANTOS, Boaventura. Descolonizar el saber, reinventar el poder. Santiago: LOM, 2013.

DEJOURS, Christophe. El sufrimiento en el trabajo. Buenos Aires: Topía. 2015.

FRENZEL, Anne. Teacher emotions. In: PEKRUN, Reinhard; LINNENBRINK-GARCIA, Lisa. (Orgs.), International Handbook of Emotions in Education. New York: Routledge. 2014, p. 494519.

FRENZEL, Anne; BECKER-KURZ, Betty; PEKRUN, Reinhard; GOETZ, Thomas. Teaching this class drives me nuts! - Examining the person and context specificity of teacher emotions. PLoS ONE, v. 10, n. 6, p. 1-16. 
FRIED, Leanne; MANSFIELD, Caroline; DOBOZY, Eva. Teacher emotion research: Introducing a conceptual model to guide future research. Issues in Educational Research, v. 25, n. 4, p. 415-441, 2015.

FULLAN, Michael. Las fuerzas del cambio, con creces. Madrid: AKAL. 2003.

HAGENAUER, Gerda; HASCHER, Tina; VOLET, Simone E. Teacher emotions in the classroom: associations with students' engagement, classroom discipline and the interpersonal teacher-student relationship. European Journal of Psychology of Education, v. 30, n. 4, 385-403, 2015.

HARGREAVES, Andy. Profesorado, cultura y postmodernidad. Cambian los tiempos, cambia el profesorado. Madrid: Morata, 1997.

HARGREAVES, Andy. The emotional practice of teaching. Teaching and Teacher Education, v. 14, n.8, p. 835- 854. 1998.

HARGREAVES, Andy. Mixed emotions: teachers' perceptions of their interactions with students. Teaching and Teacher Education, v. 16, p. 811-826, 2000.

HOCHSCHILD, Arlie Russell. The Managed Heart: Commercialization of Human Feeling. Berkeley, CA: University of California Press, 1983.

KELCHTERMANS, Geert. Teachers' emotions in educational reforms: self-understanding, vulnerable commitment and micropolitical literacy. Teaching and teacher education, $v$. 21, p. 9951006, 2005.

KELLER, Melanie, FRENZEL, Anne; GOETZ Thomas; PEKRUN, Reinhard; HENSLEY, Lauren. Exploring teacher emotions, a literature review and an experience sampling study. In: RICHARDSON, Paul; KARABENICK, Stuart; WATT, Helen. (Orgs.), Teacher motivation theory and practice. New York: Routledge, 2014, p. 69-82.

KYRIACOU, Chris. Teacher stress: Directions for future research. Educational Review, v. 53, n. 1, p. 278-295, 2001.

LASKY, Sue. A sociocultural approach to understanding teacher identity, agency and professional vulnerability in a context of secondary school reform. Teaching and teacher education, v. 21, p. 899-916, 2005.

LEE, Mikyoung; PEKRUN, Reinhard; TAXER, Jamie; SCHUTZ, Paul; VOGL, Elizabeth; XIE, Xiyao. Teachers' emotions and emotion management: integrating emotion regulation theory with emotional labor research. Social Psychology of Education, v. 19, n.4, p. 843-863, 2016.

LITTLE, Judith Warren; BARTLETT, Lora. The Teacher Workforce and Problems of Educational Equity. Review of research in education, v. 34, p. 285-328, 2010. 
MARCHANT, Teresita; MILICIC, Neva; ÁLAMOS, Pilar. Impacto en los niños de un programa de desarrollo socioemocional en dos colegios vulnerables en Chile. Revista Iberoamericana de Evaluación Educativa, v. 6, n. 2, p. 167-186, 2013.

MATURANA, Humberto. Emociones y lenguaje en educación y política. Santiago: CED, 1990.

MEVARECH, Zemira R.; MASKIT, Ditza. The teaching experience and the emotions it evokes. Social Psychology of Education, v. 18, n. 2, p. 241-253, 2015.

NARANJO, Claudio. Cambiar la educación para cambiar el mundo. Santiago: Cuarto Propio, 2007.

NEWBERRY, Melissa. Identified phases in the building and maintaining of positive teacher-student relationships. Teaching and Teacher Education, v. 26, n. 8, p. 1695-1703, 2010.

O'CONNOR, Kate Eliza. "You choose to care": teachers, emotions and professional identity. Teaching and Teacher Education, v. 24, n. 1, p. 117-126, 2008.

PEKRUN, Reinhard \& LINNENBRINK-GARCIA, Lisa. International Handbook of Emotions in Education. New York: Routledge, 2014.

POGGI, Mónica. Políticas docentes, formación, trabajo y desarrollo profesional. Buenos Aires. IIPEUNESCO, 2013.

ROCKWELL, Elsie. La experiencia etnográfica. Historia y cultura de los procesos educativos. Buenos Aires: Paidós, 2011.

ŠARIĆ, Marjeta. Teachers’ emotions: a research review from a psychological perspective. Journal of Contemporary Educational Studies, v. 66/132, n. 4, p. 10-26, 2015.

SCHLEICHER, Andreas. Teaching Excellence through Professional Learning and Policy Reform: Lessons from Around the World. Paris: OECD, 2016.

SHULMAN, Lee. Knowledge and teaching: Foundations of the new reform. Harvard Educational Review, v. 57, p. 1-22, 1987.

SCHWARTZ, Robert B; WURTZEL, Judy; OLSON, Lynn. Attracting and retaining teachers: concerns about the supply and quality of teachers are generating new policies in many OECD countries. Here's why. OECD Observer, n. 261, p. 27-29, 2007.

STAKE, Robert. The art of case study research. Thousand Oaks, CA: Sage, 1995.

Superintendencia de Seguridad Social. SUSESO-ISTAS 21. Manual de uso. Cuestionario de Evaluación de Riesgos Psicosociales en el Trabajo. Santiago: Ministerio del Trabajo de Chile, 2010. 
SUTTON, Rosemary; WHEATLEY, Karl. Teachers' emotion and teaching: A Review of the literature and directions for future research. Educational Psychology Review, v. 15, n.4, p. 327-358, 2003.

TSANG, Kwok Kuen. A Review of Current Sociological Research on Teachers' Emotions: The Way Forward. British Journal of Education, Society \& Behavioural Science, v.4, n. 2, p. 1-15, 2014.

TSANG, Kwok Kuen; Kwong, Tsun L. Emotional experience of caam in teaching: Power and interpretation of teachers' work. Frontiers in Psychology, v. 7, p. 1-11, 2016.

UITTO, Minna; JOKIKOKKO, Katri; ESTOLA, Eila. Virtual special issue on teachers and emotions in Teaching and teacher education (TATE) in 1985-2014. Teaching and Teacher Education, v. 50, p. 124-135, 2015.

UNESCO. Condiciones de trabajo y salud docente. Estudios de casos en argentina, Chile, Ecuador, México, Perú y Uruguay. Santiago: UNESCO-OREALC, 2005.

UNESCO. Tercer estudio regional comparativo y explicativo sobre logros de aprendizaje: Informe de Resultados. Santiago: OREAL-UNESCO, 2015

VAN DER DOEF, Margot; MAES, Stan. The Job-Demand-Control-(Support) Model and psychological wellbeing: a review of 20 years of empirical research. Work and Stress, v. 13, n. 5, p. 87-114, 1999.

VIGOTSKY, Lev. El desarrollo de los procesos psicológicos superiores. México: Grijalbo, 1998.

YE, Maolin; CHEN, Ye. A Literature Review on Teachers' Emotional Labor. Creative Education, v. 6, p. 2232-2240, 2015.

ZEMBYLAS, Michalinos. Emotions and Teacher Identity: A poststructural perspective. Teachers and Teaching: theory and practice, v. 9, n. 3, p. 213-238, 2003.

ZEMBYLAS, Michalinos. Discursive practices, genealogies, and emotional rules: A poststructuralist view on emotion and identity in teaching. Teaching and Teacher Education, v. 21, p. 935-948, 2005.

ZEMBYLAS, Michalinos. Emotional ecology: The intersection of emotional knowledge and pedagogical content knowledge in teaching. Teaching and Teacher Education, v. 23, n.4, p. 355367, 2007a.

ZEMBYLAS, Michalinos. Theory and methodology in researching emotions in education. International Journal of Research \& Method in Education, v.30, n. 1, p. 57-72, 2007b.

ZEMBYLAS, Michalinos; CHARALAMBOUS, Panayiota; CHARALAMBOUS, Constadina. 
Manifestations of Greek-Cypriot teachers' discomfort toward a peace education initiative: engaging with discomfort pedagogically. Teaching and Teacher Education, v.28, n. 8, p. 1071-1082, 2012.

ZEMBYLAS, Michalinos; SCHUTZ, Paul (Orgs). Methodological advances in research on education and emotion. Switzerland: Spinger International Publishing, 2016.

ZEMBYLAS, Michalinos; \& SCHUTZ, Paul. (Orgs) Advances in teacher emotion research. The impact on teachers' lives. London: Springer, 2009.

ZEMELMAN, Hugo. Los horizontes de la razón. Uso crítico de la teoría. México: Editorial Anthropos, Colegio de México, 1992.

\section{Correspondência}

Rodrigo Cornejo Chávez: Professor Associado do Departamento de Psicologia da Universidade do Chile. E-mail: rodrigo.cornejo@uchile.cl

Gabriel Etcheberrigaray: Programa Equipo Psicología Educación y Sociedad. Universidad de Chile E-mail: garay89@gmail.com

Sebastián Vargas: Programa Equipo Psicología Educación y Sociedad. Universidad de Chile E-mail: seba.vargasp@gmail.com

Jenny Assaél: Professora Titular do Departamento de Psicologia da Universidade do Chile. E-mail: jassael@uchile.cl

Texto publicado em Currículo sem Fronteiras com autorização dos autores 